\title{
PScan 1.0: flexible software framework for polygon based multiphoton microscopy
}

\author{
Yongxiao Li ${ }^{\mathrm{a}}$, Woei Ming Lee*a

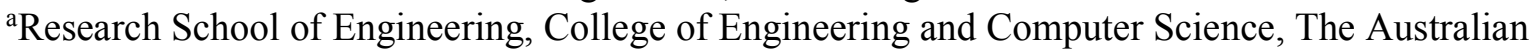 \\ National University, 31 North Road, Canberra, ACT, Australia 2601
}

\begin{abstract}
Multiphoton laser scanning microscopes exhibit highly localized nonlinear optical excitation and are powerful instruments for in-vivo deep tissue imaging. Customized multiphoton microscopy has a significantly superior performance for in-vivo imaging because of precise control over the scanning and detection system. To date, there have been several flexible software platforms catered to custom built microscopy systems i.e. ScanImage, HelioScan, MicroManager, that perform at imaging speeds of 30-100fps. In this paper, we describe a flexible software framework for high speed imaging systems capable of operating from $5 \mathrm{fps}$ to $1600 \mathrm{fps}$. The software is based on the MATLAB image processing toolbox. It has the capability to communicate directly with a high performing imaging card (Matrox Solios eA/XA), thus retaining high speed acquisition. The program is also designed to communicate with LabVIEW and Fiji for instrument control and image processing. Pscan 1.0 can handle high imaging rates and contains sufficient flexibility for users to adapt to their high speed imaging systems.
\end{abstract}

Keywords: Multiphoton microscopy, control software, MATLAB, polygon mirror

\section{INTRODUCTION}

Multiphoton laser scanning microscopy (MPLSM) is one kind of nonlinear optical microscope which has been widely used in intravital tissue observation ${ }^{1}$. With the ability of focusing both on the temporal and spatial domain, MPLSM provides deep tissue intravital sub-micro resolution imaging, especially for neuroscience. For high imaging speed, polygon mirror scanning microscope is a good choice with its rotation speed range and broad scanning angle ${ }^{2}$.

Customized control software for multiphoton microscopies are also developed, such as ScanImage, HelioScan, MPscope and Micro-Manager ${ }^{3,4,5,6}$. These four softwares are written in different computer languages and compile environments, thus have different features and application orientations. In detail, ScanImage is written by MATLAB exclusively, it requires the configuration to start. It has been widely used by microscopists who built their own microscope. HelioScan is run by LabVIEW, which has a series packages for different imaging modes. MPscope is written by Object Pascal for fast frame rate concerning of multiphoton microscope. MicroManager is Java based combined with the ImageJ software, which is popular for biology image post processing. All of them have their own advantages and drawbacks, popular language and efficient speed, complexity and multiple functions, component price and open source, all are constrained to each other ${ }^{7,8}$.

To solve these problems, we developed a customized software called PScan 1.0, which is MATLAB based considering its popularity in biology area and image matrix calculation. PScan 1.0 is designed for the polygon mirror based laser scanning multiphoton microscope. For a full utilization of other language advantages, PScan 1.0 uses ActiveX technology to call the LabVIEW program to control the PCI-6110 card from National Instruments. Use Fiji as the MATLAB-Java interface to call the ImageJ software. Use script language to dynamically change the frame grabber. All these characters maintain PScan 1.0 to be a simple control software which requires little knowledge and configuration to use it while still has a high speed compares with other software. PScan 1.0 is developed for filling the gap between polygon mirror laser scanning microscope and computer control.

*steve.lee@anu.edu.au

SPIE BioPhotonics Australasia, edited by Mark R. Hutchinson, Ewa M. Goldys, Proc. of SPIE Vol. 10013, 1001333 - @ 2016 SPIE · CCC code: 0277-786X/16/\$18 · doi: 10.1117/12.2242954 


\section{METHODS}

\subsection{PScan 1.0 schematic}

PScan 1.0 is MATLAB based with user interfaces to other software environment. Its constitution is shown in figure 1 . It offers the functionalities of scanning control, image preview and acquisition, real-time image processing and data management as well as post manipulation.

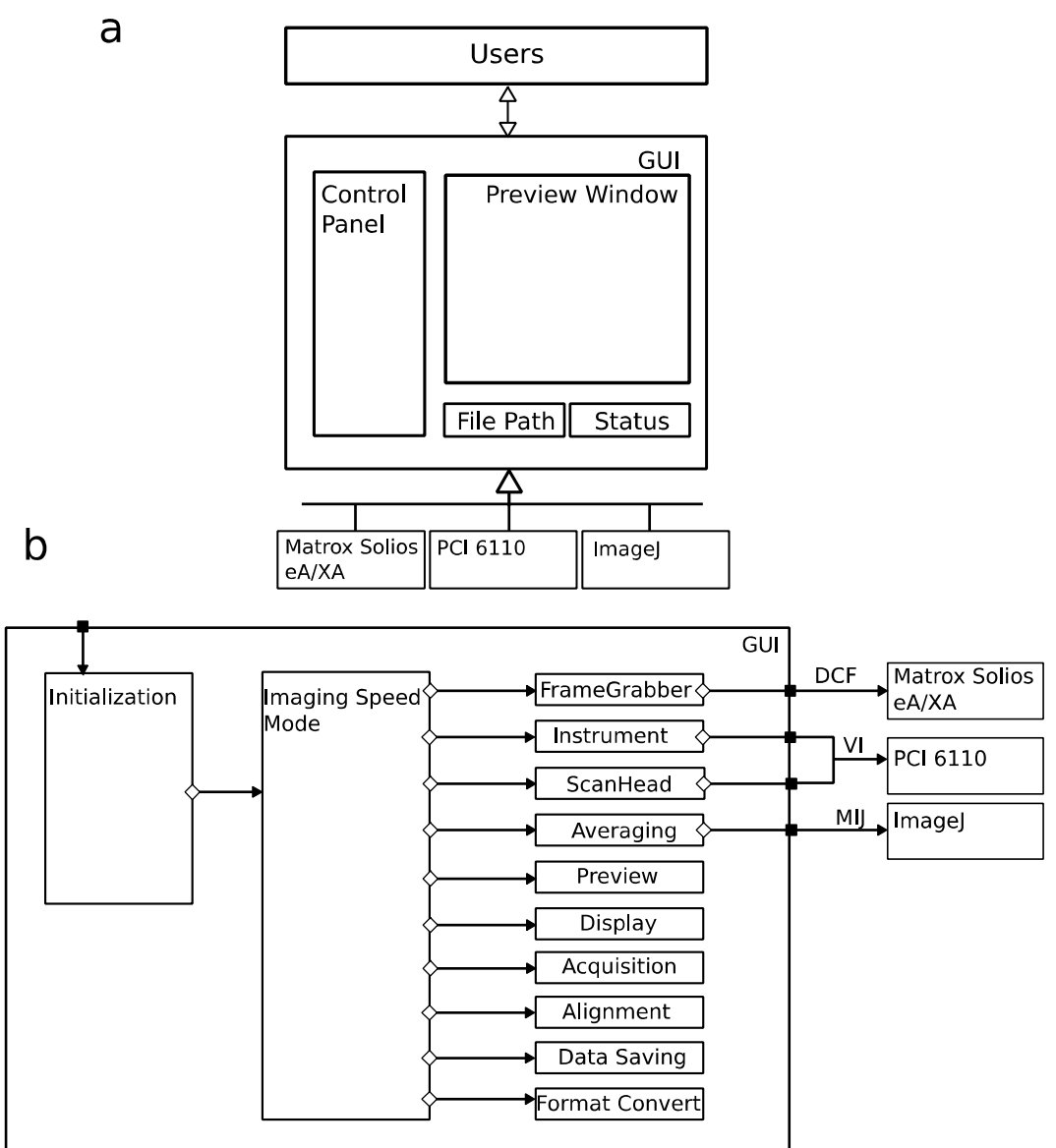

Figure 1. Schematic view of PScan 1.0. (a) Top view of the PScan 1.0 with its interfaces among the other language environments. (b) Detail illustration of combined components inside PScan 1.0.

The user interface GUI of PScan 1.0 is quite simple and easy to use, which is shown in figure 2. While at the same, PScan 1.0 maintained several key functions. The preview window has red channel, green channel, combined channel and averaged channel. The acquisition panel includes the preview, acquisition, alignment, saving and averaging functions. It also has the color reference panel, region of interest panel (ROI), acquisition timing panel, scanning area channel, imaging size channel and speed mode channel. All the working status is indicated at the bottom left corner, users can know how is the progressing of PScan 1.0. 


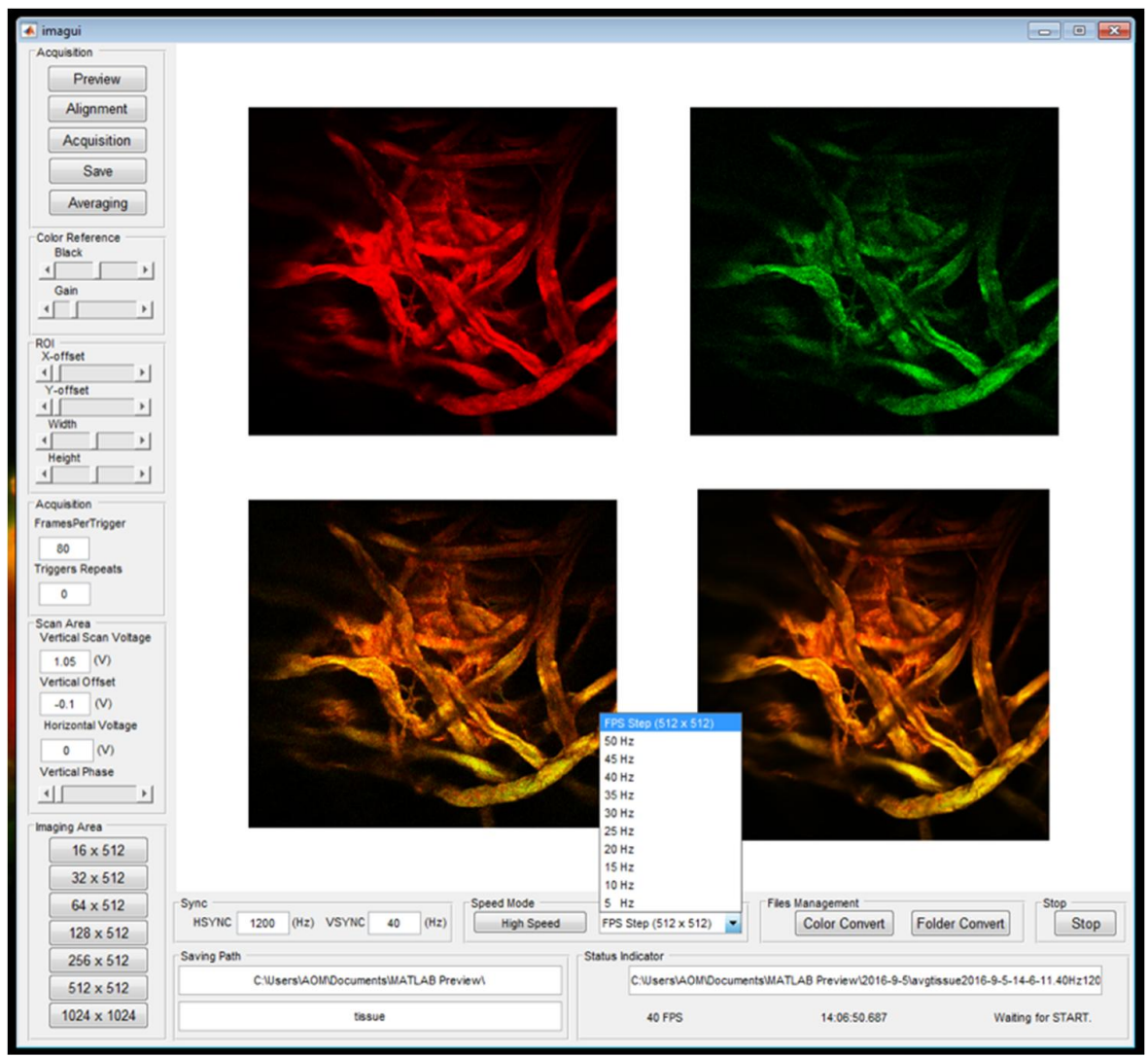

Figure 2. GUI of PScan 1.0. The 'Acquisition' panel is at the upper left corner, which contains the manipulations of image acquisition, alignment and saving as well as averaging. Beneath the acquisition is the parameter setting panels of color, ROI and duration. Then next is the panel of scanning area control, which is used to control the two galvanometer mirrors. At the lower left corner is the image format, a controlling of the frame grabber. The image channels are red, green, combined dual color channel and averaged image channel respectively. Beneath the image channels are synchronizing control and speed mode options as well as the post data management. At the lower right corner are the saving path text bar and status indicator.

\subsection{PScan 1.0 registration for in-vivo imaging}

Respiratory and breath would result in motion and vibrations during in-vivo imaging, which need to be eliminated for following data processing such as the blood flow measurement, otherwise the accuracy is damaged. Here we utilize a subpixel registration algorithm for live-preview image alignment and post image registration in PScan 1.0. The algorithm is based on the centroid weight algorithm, with a precision from 0.01 to 0.1 pixel decided by the image $\mathrm{SNR}^{8}$. 


\section{a}
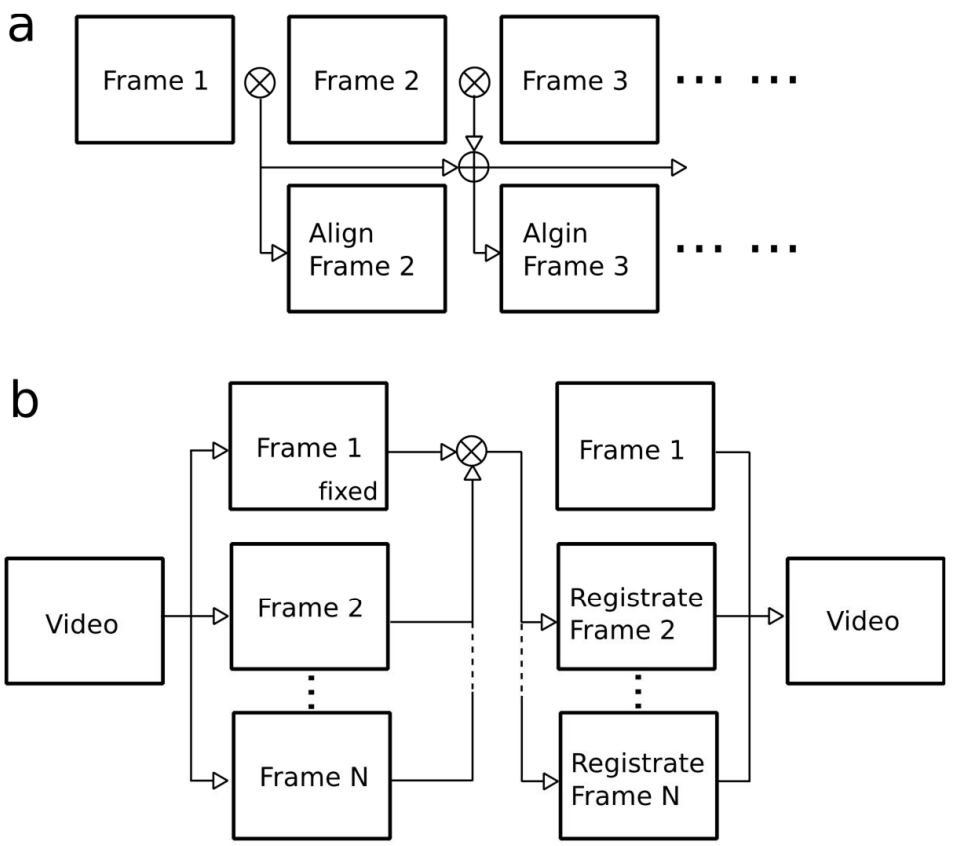

C

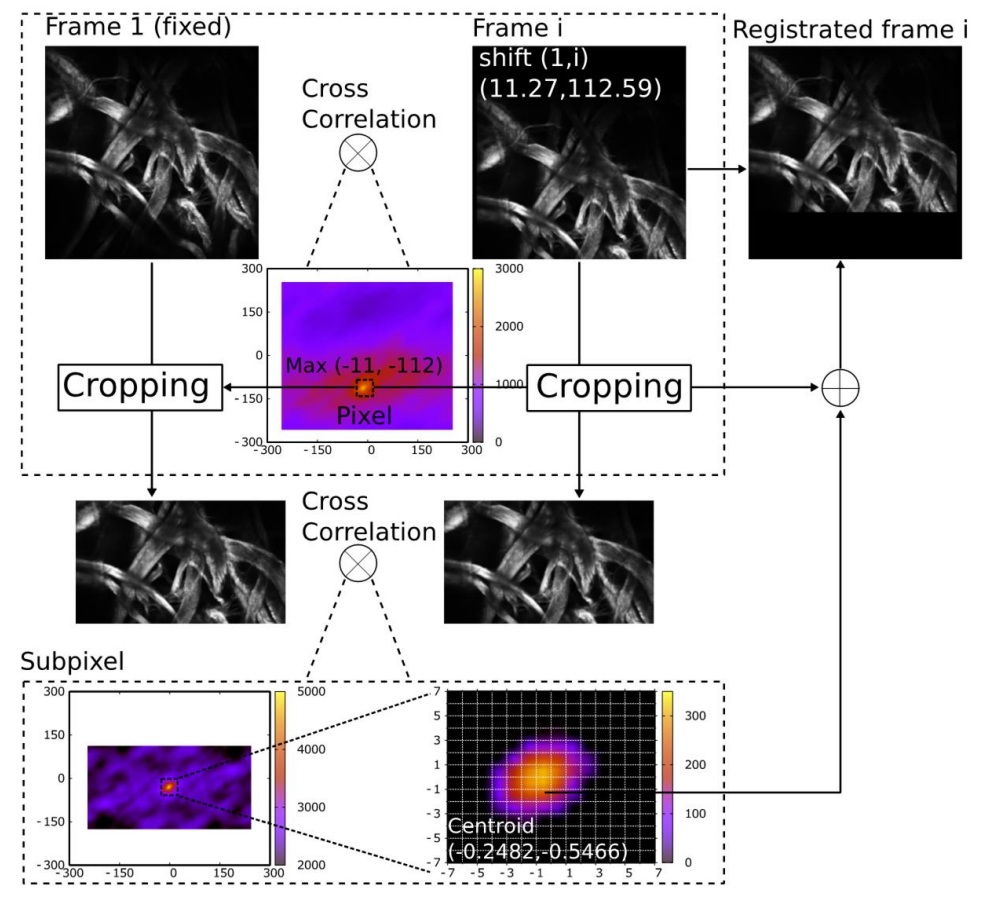

Figure 3. Sub pixel registration algorithm of PScan 1.0. (a) The schematic used in live-preview. (b) The schematic of subpixel registration used in post processing. (c) The algorithm theory chart, through double cross correlation algorithm with mesh grid centroid measurement. 


\section{RESULTS}

Figure 4 is taken from an in-vivo mouse imaging experiment by a PScan 1.0 controlled polygon mirror laser scanning multiphoton microscope. It is the image of microvasculature of a wild type mouse with fluorescence dextran-rhodamine dye. Figure 4 shows the averaged results of raw data, live preview aligned data and the post processed data. Blurs in averaged image are decreasing with the increase of registration precision level.
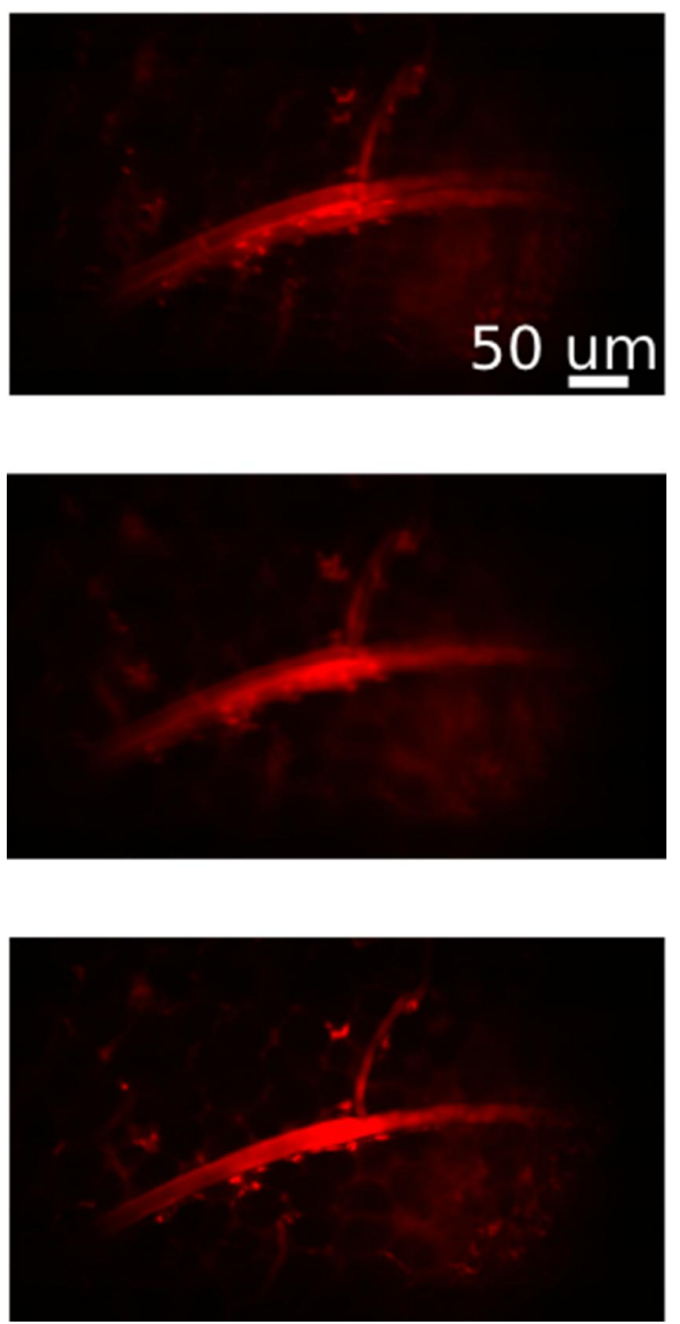

Figure 4. Averaged image of in-vivo mouse vessel organ. From top to bottom are raw data, live preview alignment processing and subpixel registration post processing.

\section{CONCLUSION}

PScan 1.0 is currently developed and run on Windows based computer with Matrox frame grabber and National Instrument cards. In the furture, we hope to add more supports to different cards. And also deploy the GPU based image processing 
modules, such as neuron signal processing and other types of scanning strategy. And most importantly, keep PScan's imaging speed as fast as we can while maintaining the user interface as easy as we could.

\section{REFERENCES}

[1] Denk, W., Strickler, J. H. and Webb, W. W., "Two-photon laser scanning fluorescence microscopy," Science 248(4951), 73-76 (1990).

[2] Israel, V., Joel, A. S., David, P. B., Daniel, C. and Charles, P. L., "In Vivo Cell Tracking with Video Rate Multimodality, " IEEE Journal of Selected Topics in Quantum Electronics 14(1), 10-18 (2008).

[3] Thomas, A. P., Bernado, L. S. and Karel S., "ScanImage: Flexible software for operating laser scanning microscopes," Biomedical Engineering Online, 2(1), 1-9 (2003).

[4] Dominik, L., Marcel, V. H., Andreas, J. K., Chetan, N., Oliver, A. P., Maurice, G., Hansjorg, K. and Fritjof, H., "HelioScan: A software framework for controlling in vivo microscopy setups with high hardware flexibility, functional diversity and extendibility, "Journal of NeuroScience Methods 215(1), 38-52 (2013).

[5] Arthur, E., Mark, T., Nenad A., Henry, P., Ronald, V. and Nico, S., "Advanced methods of microscope control using $\mu$ Manager software," Journal of Biological Methods 1(2), 2014.

[6] Quoc, T. N., Philbert, S. T. and David, K., "MPScope: A versatile software suite for multiphoton microscopy," Journal of Neuroscience Methods 156(1-2), 351-359 (2006).

[7] Kevin, W. E., Michael R. B., Ilya, G. G., Luis, I., B. S., Manjunath, Maryann, E. M., Robert, F. M., Hanchuan, P., Anne, L. P., Badrinath, R., Nico, S., Jason, R. S., Pavel, Tomancak and Anne, E. C., "Biological imaging software tools," Nature Methods 9(7), 697-710 (2012).

[8] Kelly, R. C., "Imaging and detection: focusing on software," Nature Methods 5(7), 651-658 (2008).

[9] Song, F., Linhua, D., Guofeng, S., Feng, W., Hui, D. and Kaifan, J., "A Subpixel Registration Algorithm for Low PSNR Images," Proc. IEEE ICACI, 626-630 (2012). 